\title{
Enhanced noninvasive imaging of oncology models using the NIS reporter gene and bioluminescence imaging
}

\author{
Rianna Vandergaast ${ }^{1} \cdot$ Sarawut Khongwichit ${ }^{2,3} \cdot$ Huailei Jiang $^{4} \cdot$ Timothy R. DeGrado $^{4} \cdot$ Kah-Whye Peng $^{1,2} \cdot$ \\ Duncan R. Smith $\mathbb{1}^{3}$. Stephen J. Russell ${ }^{1,2} \cdot$ Lukkana Suksanpaisan $^{1}$
}

Received: 1 November 2018 / Revised: 11 December 2018 / Accepted: 28 December 2018 / Published online: 24 January 2019

(c) The Author(s) 2019. This article is published with open access

\begin{abstract}
Noninvasive bioluminescence imaging (BLI) of luciferase-expressing tumor cells has advanced pre-clinical evaluation of cancer therapies. Yet despite its successes, BLI is limited by poor spatial resolution and signal penetration, making it unusable for deep tissue or large animal imaging and preventing precise anatomical localization or signal quantification. To refine pre-clinical BLI methods and circumvent these limitations, we compared and ultimately combined BLI with tomographic, quantitative imaging of the sodium iodide symporter (NIS). To this end, we generated tumor cell lines expressing luciferase, NIS, or both reporters, and established tumor models in mice. BLI provided sensitive early detection of tumors and relatively easy monitoring of disease progression. However, spatial resolution was poor, and as the tumors grew, deep thoracic tumor signals were massked by overwhelming surface signals from superficial tumors. In contrast, NISexpressing tumors were readily distinguished and precisely localized at all tissue depths by positron emission tomography (PET) or single photon emission computed tomography (SPECT) imaging. Furthermore, radiotracer uptake for each tumor could be quantitated noninvasively. Ultimately, combining BLI and NIS imaging represented a significant enhancement over traditional BLI, providing more information about tumor size and location. This combined imaging approach should facilitate comprehensive evaluation of tumor responses to given therapies.
\end{abstract}

\section{Introduction}

Early evaluation of cancer therapies relies heavily on rodent oncology models to establish the effects of potential treatments on tumor growth and metastases. In the last several decades, noninvasive imaging of reporter geneexpressing tumor cells has become an important

Supplementary information The online version of this article (https:// doi.org/10.1038/s41417-019-0081-2) contains supplementary material, which is available to authorized users.

Lukkana Suksanpaisan

suksanpaisan@imanislife.com

Imanis Life Sciences, Rochester, MN 55902, USA

2 Department of Molecular Medicine, Mayo Clinic, Rochester, MN 55905, USA

3 Institute of Molecular Biosciences, Mahidol University, Salaya, Nakhon Pathom 73170, Thailand

4 Deparment of Radiology, Mayo Clinic, Rochester, MN 55905, USA technology for pre-clinical oncology studies, providing researchers with a way to track tumor responses to therapies in real-time, longitudinally, in the same animal [1-3]. These studies have predominately utilized optical reporter genes, including firefly luciferase (Fluc) and a variety of fluorescent proteins (FPs). In particular, bioluminescence imaging (BLI) of Fluc has become popular due to its relative ease-of-use and the high sensitivity that it affords [4, 5]. Nevertheless, BLI suffers from poor spatial resolution and limited signal penetration, which reduce the depth of information that can be acquired using BLI [5]. Here, we sought to enhance and refine tumor imaging by combining BLI with high-resolution, tomographic single photon emission computed tomography (SPECT) or positron emission tomography (PET) imaging using the sodium iodide symporter (NIS) reporter gene.

NIS is a transmembrane protein that mediates uptake of iodide and several other anions into cells. The protein is endogenously expressed in the thyroid - where its activity is critical for normal synthesis of thyroid hormones-and to a lesser degree in the gastric mucosa, salivary glands, and lactating mammary glands [6-10]. Importantly for imaging, 
NIS mediates the uptake of several clinically approved radiotracers. Therefore, cells engineered to express NIS, either directly or as a result of infection with a NISexpressing vector, can be tracked noninvasively by SPECT or PET imaging [9-13]. NIS has been used to track or assess regenerative cells [14-19], cellular therapies [20,21], immune cells [22-24], viral vectors [25], oncolytic viruses $[11,26-30]$, and tumor cells [31-33] in small and large animal models as well as in humans. Moreover, the versatility of NIS to concentrate multiple readily available SPECT and PET radiotracers facilitates its widespread adoption in pre-clinical imaging.

In this study, we used several oncology models to compare BLI and NIS imaging modalities. Many of our studies used 4T1 cells, which are a highly metastatic model for triple-negative stage IV breast cancer, and are frequently tracked noninvasively using BLI. When implanted into immunocompromised mice or syngeneic Balb/c mice, 4T1 cells form spontaneous metastases at multiple sites including the lungs, liver, lymph nodes, bone, and CNS $[34,35]$, making them an ideal model for comparing imaging modalities at different tissue depths and anatomical locations. We demonstrate here that combination BLI and NIS imaging significantly increases the precision and accuracy of in vivo tumor characterization, while maintaining sensitive and relatively easy tumor detection and monitoring. This dual reporter imaging approach has the potential to enhance pre-clinical studies of cancer therapies by providing the ultimate clarity on tumor responses, while reducing animal use and study costs.

\section{Materials and methods}

\section{Generation of reporter gene cell lines}

Parental tumor cell lines were purchased from ATCC and maintained at $37^{\circ} \mathrm{C} / 5 \% \mathrm{CO}_{2}$ in the appropriate media supplemented with $10 \%$ fetal bovine serum (Life Technologies, Carlsbad, CA, USA) and $1 \times$ Pen/Strep. Human lung adenocarcinoma A549 cells (ATCC ${ }^{\circ}$ CCL-185 $5^{\mathrm{TM}}$ ) and murine Lewis Lung carcinoma LL/2 cells (ATCC ${ }^{\circ}$ CRL-1642 ${ }^{\mathrm{TM}}$ ) were grown in Dulbecco's modification of Eagle medium (Life Technologies), murine mammary carcinoma 4T1 cells (ATCC $^{\circ}$ CRL-2539 $9^{\mathrm{TM}}$ ) were grown in RPMI-1640 (Life Technologies), and human lymphoma Nalm6 cells (ATCC CRL-3273 ${ }^{\mathrm{TM}}$ ) were grown in RPMI-1640 supplemented with an additional $10 \mathrm{~mm}$ 4-(2-hydroxyethyl)-1-piperazineethanesulfonic acid (HEPES) (Mediatech, Manassas, VA, USA). All parental cell lines were authenticated by short tandem repeat profiling (IDEXX BioResearch, Columbia, MO, USA). Tumor cell lines stably expressing reporter genes were generated by lentiviral vector transduction. The following lentiviral vectors from Imanis Life Sciences (Rochester, MN, USA) were used: LV-Fluc-P2A-Neo (LV011), LV-Fluc-P2A-Puro (LV012), LV-hNIS-IRES-Neo (LV013), LV-mNIS (LV008), LV-mNIS-PGK-Puro (LV022), and LV-Luc2-P2A-hNIS (LV023). Cells were transduced at a multiplicity of infection of 10 in the presence of $8 \mu \mathrm{g} / \mathrm{ml}$ polybrene. Cells transduced with lentiviral vector containing a neomycin (Neo) or puromycin (Puro) resistance gene were selected using G418 or puromycin, respectively. The A549-hNIS-Neo/Fluc-Puro (CL083) and 4T1-Fluc-Neo/ mNIS-Puro (CL066) cell lines were generated by subsequent transduction with two separate lentiviral vectors. The LL/2mNIS (CL114), 4T1-Fluc-Neo (CL126), 4T1-mNIS (CL117), and Nalm6-Fluc-hNIS (CL143) cell lines underwent clonal selection using ClonaCell TCS (Stemcell Technologies, Vancouver, Canada). All of the generated oncology cell lines tested negative for mycoplasma and are available commercially at Imanis Life Sciences.

\section{In vitro luciferase assays}

A549-hNIS-Neo/Fluc-Puro or parental A549 cells were seeded at densities of 2 through $1 \times 10^{6}$ cells/well in 96-well plates. D-luciferin (Gold Biotechnology, St. Louis, MO, USA) was added to wells at a final concentration of $3 \mathrm{mg} / \mathrm{ml}$ and luminescence was immediately measured on an Xenogen IVIS Spectrum (Perkin Elmer, Waltham, MA, USA). The total flux (photons $/ \mathrm{s} / \mathrm{cm}^{2} / \mathrm{sr}$ ) of wells was determined using Living Image software (Perkin Elmer). Values represent the mean $( \pm$ standard error) total flux from three independent experiments performed in triplicate.

\section{In vitro NIS ${ }^{125}$ I uptake assay}

A549-hNIS-Neo/Fluc-Puro cells were seeded in 6-well plates at densities of 10 through $1 \times 10^{6}$ cells/well. To maintain cell viability, parental A549 cells were added to each well so that the total cell density in each well was constant at $1 \times 10^{6}$ cells/ well. Cells were allowed to attach overnight and then subject to an ${ }^{125}$ I uptake assay. Briefly, cells were washed once with $10 \mathrm{mM}$ HEPES in Hank's Balanced Salt Solution (HEPES/ HBSS) and incubated with ${ }^{125} \mathrm{I}$ in HEPES/HBSS at $37^{\circ} \mathrm{C}$. After $1 \mathrm{~h}$, cells were washed twice with cold HEPES/HBSS and lysed in $1 \mathrm{M}$ sodium hydroxide. Uptake of ${ }^{125} \mathrm{I}$ in the cell lysates was quantitated (in CPM) using an ISO Data-10 gamma counter (GMI Inc., Ramsey, MN 55303). Values represent the mean ( \pm standard error) ${ }^{125} \mathrm{I}$ uptake in CPM from three independent experiments performed in triplicate.

\section{In vitro NIS $\left[{ }^{18} \mathrm{~F}\right]-\mathrm{TFB}$ uptake assay}

Increasing numbers of A549-hNIS-Neo/Fluc-Puro cells were mixed with parental A549 cells in microcentrifuge 
tubes, such that each tube contained a total of $1 \times 10^{6}$ cells. The cells were incubated at $37{ }^{\circ} \mathrm{C}$ for $30 \mathrm{~min}$ with $2 \mu \mathrm{Ci}\left[{ }^{18} \mathrm{~F}\right]$-TFB in $1 \mathrm{ml}$ of HEPES/HBSS. The cells were centrifuged and washed once with cold HEPES/ HBSS. The final cell pellets were immediately imaged on a small animal Inveon Multiple Modality PET/CT scanner (Siemens Medical Solutions USA, Inc., Malvern, PA, USA). CT was performed at $60 \mathrm{kEv}, 500 \mu \mathrm{A}$, with 400 ms/projection, 180 projections, and bin 4 . The effective pixel size was $94.26 \mu \mathrm{m}$. PET was performed at $10 \mathrm{~min}$ acquisition, 3DRP reconstruction with Colsher filter, and 0.5 cutoff. Co-registered images were rendered and visualized using the PMOD software (PMOD Technologies, Zurich, Switzerland). Values represent the mean ( \pm standard deviation) $\left[{ }^{18} \mathrm{~F}\right]-\mathrm{TFB}$ uptake in $\mu \mathrm{Ci}$.

\section{Establishment of in vivo tumor models}

All animal procedures were reviewed and approved by the Mayo Clinic Institutional Animal Care and Use Committee. Balb/c (6-week, female), C57Bl/6 (6-week, female), and Fox SCID beige (6-week, male and female) mice were purchased from Envigo (Indianapolis, IN, USA) or Charles River (Wilmington, MA, USA). Prior to implantation, cells were washed once with cold $\left(4{ }^{\circ} \mathrm{C}\right)$ PBS and resuspended in cold PBS at an appropriate concentration based on the route of implantation. To establish the LL/2, 4T1, and Nalm6 experimental metastases models, mice were implanted with $1 \times 10^{6}$ cells via tail vein injection. To establish subcutaneous tumors, mice $(=5)$ were implanted in the right hind flank with $2 \mathrm{x}$ $10^{6}$ cells. Tumor growth and metastases were monitored by BLI and NIS imaging. Tumor volumes were determined by calipers or CT. For caliper determinations, the length and width of each tumor was measured by caliper, and the tumor volume was calculated using the equation $a^{2} \times(b / 2)$, where $a$ is the shortest dimension. In other cases, tumor area was defined based on CT image using PMOD software.

\section{Noninvasive BLI}

Mice received an intraperitoneal injection ( $3 \mathrm{mg} / \mathrm{mouse}$ ) of d-luciferin $10 \mathrm{~min}$ before imaging. Bioluminescent signal and grayscale photographic images were acquired using a Xenogen IVIS Spectrum instrument and Living Image software. During image acquisition, mice were maintained under general anesthesia with isoflurane. Bioluminescent signal quantification (photons $/ \mathrm{s} / \mathrm{cm}^{2} / \mathrm{sr}$ ) of regions of interest was carried out using Living Image software. Individual images from different time points were cropped and complied using Adobe Photoshop
Elements and Adobe Illustrator (Adobe Inc., San Jose, CA, USA).

\section{Nuclear imaging}

For SPECT imaging, mice were injected with $300 \mu \mathrm{Ci}$ of $\left[{ }^{99 \mathrm{~m}} \mathrm{Tc}\right]-$ pertechnetate via tail vein $1 \mathrm{~h}$ prior to image acquisition. Imaging was performed in the Mayo Clinic Small Animal Imaging Core Facility using a U-SPECT-II/ CT scanner (MILabs, Utrecht, The Netherlands). Scan volumes for both the SPECT and CT were selected based on orthogonal optical images provided by integrated webcams. Micro-CT image acquisition was performed in $4 \mathrm{~min}$, for normal resolution (169- $\mu \mathrm{m}$ square voxels, 640 slices) at $0.5 \mathrm{~mA}$ and $60 \mathrm{kV}$. Image acquisition time was $\sim 20 \mathrm{~min}$ for SPECT (69 projections at 50 seconds per bed position). All pinholes focused on a single volume in the center of the tube; by using an XYZ stage, large volumes up to the entire animal were scanned at uniform resolution [36]. Coregistration of the SPECT and CT images was performed by applying pre-calibrated spatial transformation to the SPECT images to match with the CT images. SPECT reconstruction was performed using a POSEM (pixel-based ordered subset expectation maximization) algorithm [37] with six iterations and 16 subsets. CT data were reconstructed using a Feldkamp cone beam algorithm (NRecon v1.6.3, Skyscan). After reconstruction, SPECT images were automatically registered to the $\mathrm{CT}$ images according to the pre-calibrated transformation, and re-sampled to the CT voxel size. Coregistered images were further rendered and visualized using the PMOD software. A 3D-Guassian filter $(0.8 \mathrm{~mm}$ full-width at half maximum) was applied to suppress noise, and LUTs (Look Up Tables) were adjusted for good visual contrast. Reconstructed images were visualized as both orthogonal slices and maximum intensity projections. Maximal intensity projection videos and three-dimensional renderings of regions of interests were performed on the PMOD software.

For PET imaging, mice received $300 \mu \mathrm{Ci}$ of $\left[{ }^{18} \mathrm{~F}\right]-\mathrm{TFB}$ [38] 45 min prior to image acquisition. PET/CT imaging was performed on a small animal Inveon Multiple Modality PET/CT scanner. CT was performed at $80 \mathrm{kEv}$, $500 \mu \mathrm{A}$, with $250 \mathrm{~ms} /$ projection, 180 projections, and bin 4 ; the effective pixel size was $94.59 \mu \mathrm{m}$. PET was performed using $10 \mathrm{~min}$ acquisition, OSEM2D reconstruction with Fourier rebinning, and four iterations. Co-registered images were rendered and visualized using the PMOD software.

In order to improve tumor visualization, signals in the thyroid, salivary glands, and stomach owing to endogenous NIS, and in the bladder owing to secreted radiotracer were removed from images using PMOD software. 

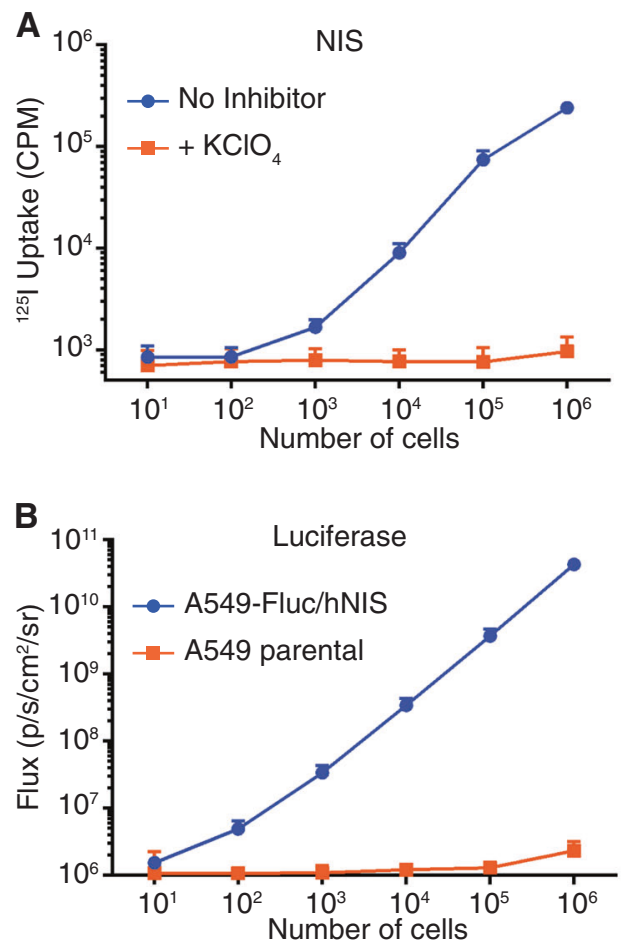

Fig. 1 In vitro reporter activity of A549-hNIS-Neo/Fluc-Puro cells. a The indicated number of A549-hNIS-Neo/Fluc-Puro cells were seeded in six-well plates and an ${ }^{125} \mathrm{I}$ uptake assay was performed in the presence or absence of the NIS inhibitor $\mathrm{KClO}_{4}$. b The indicated number of A549-hNIS-Neo/Fluc-Puro or parental A549 cells were seeded in 96-well plates, and luminescence (Flux) was measured immediately after the addition of $3 \mathrm{mg} / \mathrm{ml}$ d-luciferin. c, d The indicated number of

\section{Results}

\section{NIS radiotracer uptake correlates with cell number}

The main objective of this study was to define a method for improved accuracy and precision in pre-clinical tumor imaging by using the NIS reporter to enhance standard BLI. To this end, we first generated tumor cell lines expressing NIS and firefly luciferase (Fluc), and characterized NIS and luciferase signal in vitro. NIS activity correlated strongly with cell number in vitro, except at very low numbers of NIS-expressing cells (Fig. 1a). Fluc activity also correlated strongly to cell number over a wide cell range (Fig. 1b). To determine the in vitro sensitivity of NIS imaging by PET, uptake of radiotracer $\left[{ }^{18} \mathrm{~F}\right]$-TFB in cell pellets of NISexpressing cells was imaged using PET. For these experiments, each cell pellet contained a total of $1 \times 10^{6}$ cells, but the number of NIS-expressing cells was increased from $1 \times$ $10^{3}$ to $1 \times 10^{6}$ cells. The cells were incubated with $\left[{ }^{18} \mathrm{~F}\right]$ TFB for $30 \mathrm{~min}$, before being washed and pelleted for imaging. $\left[{ }^{18} \mathrm{~F}\right]$-TFB uptake directly correlated with cell number from $1 \times 10^{3}$ to $1 \times 10^{6}$ cells (Fig. $1 \mathrm{c}$ and data not shown). Although we detected 1000 NIS-expressing cells in one tube, 2500 NIS-expressing cells was required for

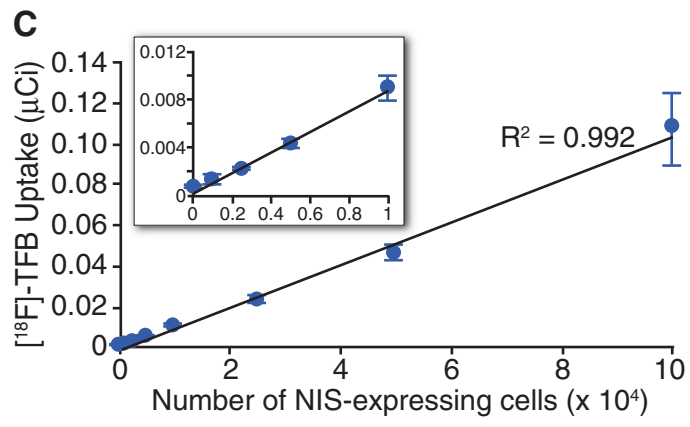

D
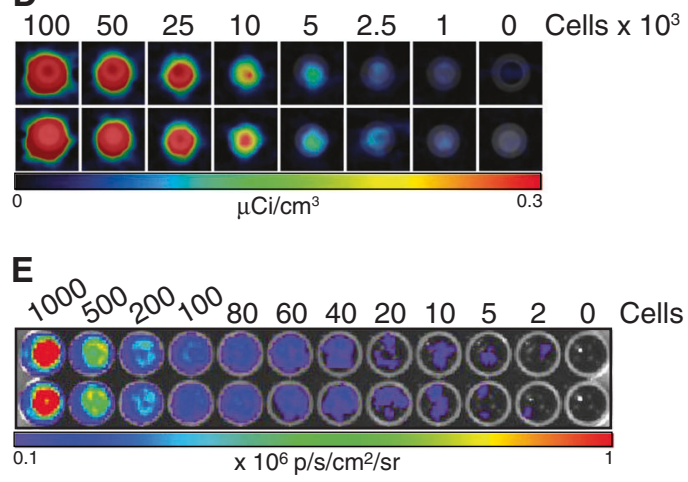

A549-hNIS-Neo/Fluc-Puro cells were mixed with parental A549 cells to a total of $1 \times 10^{6}$ cells/tube and incubated for $30 \mathrm{~min}$ with $\left[{ }^{18} \mathrm{~F}\right]$-TFB. Following a wash to remove residual $\left[{ }^{18} \mathrm{~F}\right]-\mathrm{TFB}$, the cell pellets were imaged by PET/CT $\mathbf{d}$ and the $\left[{ }^{18} \mathrm{~F}\right]$-TFB uptake from each tube was determined c. e The indicated number of A549-hNIS-Neo/Fluc-Puro cells were seeded in 96-well plates, and BLI was performed immediately after the addition of $3 \mathrm{mg} / \mathrm{ml} \mathrm{D}-$ luciferin

consistent detection (Fig. 1d), indicating the sensitivity of PET imaging was approximately 2500 NIS-expressing cells in a cell pellet of $1 \times 10^{6}$ cells. By comparison, as few as 2 Fluc-expressing cells could be consistently detected using BLI (Fig. 1e). Taken together, these findings demonstrated that reporter signal intensity is an accurate indicator of cell number for both NIS and luciferase over a wide cell range. Moreover, both BLI and NIS imaging offer sensitive detection of reporter cells. Although luciferase offers superior sensitivity in vitro, it is subject to tissue attenuation, which can rapidly reduce sensitivity in vivo at increased tissue depths.

\section{High-resolution NIS imaging facilitates precise tumor localization for discrimination of deep-tissue tumors}

Although BLI is frequently used to noninvasively image tumor models, precise imaging and localization of tumor metastases is difficult using BLI due to poor spatial resolution and tissue attenuation of signal that results in surfaceweighted images. In contrast, NIS imaging by small animal SPECT or PET produces tomographic images with spatial resolution below 1-mm, dependent on the device used for 

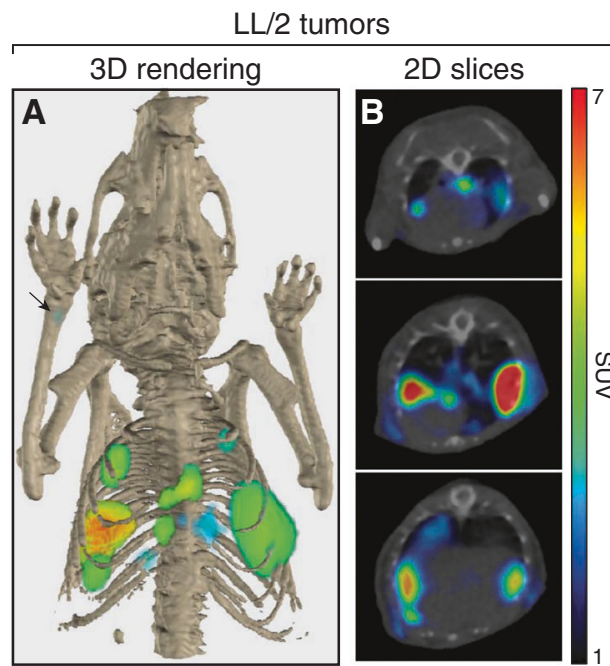

Fig. 2 High-resolution tumor imaging with precise anatomical localization using NIS. a, b C57B1/6 mice were implanted intravenously with LL/2-mNIS cells; a representative mouse is shown. LL/2-mNIS tumors were imaged by PET/CT using $\left[{ }^{18} \mathrm{~F}\right]-\mathrm{TFB}$ on day 27 . Shown is a $3 \mathrm{D}$ rendering of tumor localization within the mouse (arrow indicates

image acquisition $[39,40]$. As a proof of concept, we implanted mice with murine Lewis lung carcinoma LL/2 cells expressing NIS (LL/2-mNIS) and acquired highresolution tomographic images of the tumors within the mice (Fig. 2a, b). Precise anatomical localization of NIS signal was possible through the combination of PET imaging with X-ray computed tomography (CT). Tumors were primarily observed within the thoracic cavity, though one small bone metastasis was also detected (Fig. 2a; arrow). Precise anatomical localization of NIS reporter signal was also clearly demonstrated in mice implanted with acute lymphoblastic leukemia Nalm6 cells expressing luciferase and NIS (Nalm6-Fluc-hNIS). BLI of these mice (Fig. 2c) revealed a tumor growth pattern consistent with the expected spinal and bone metastases [41-44]. Using SPECT/CT imaging of NIS, we precisely and definitively localized the tumors to the long bones noninvasively (Fig. 2d and S1). NIS imaging, therefore, provided an important level of accuracy and precision for tumor imaging that could not be obtained with BLI alone.

The importance of high spatial resolution combined with precise anatomical localization for tumor imaging was further demonstrated when we imaged mice with mammary carcinoma 4T1 tumors. A significant global tumor burden was detected in mice implanted with luciferase-expressing 4T1-Fluc cells (Fig. 3a), though precise localization was not possible. Moreover, superficial luciferase signals from subcutaneous tumors masked the deep-tissue thoracic signals. Little or no luciferase signal was observed in the lungs of killed mice (Fig. 3b), though numerous tumors were readily detected by BLI

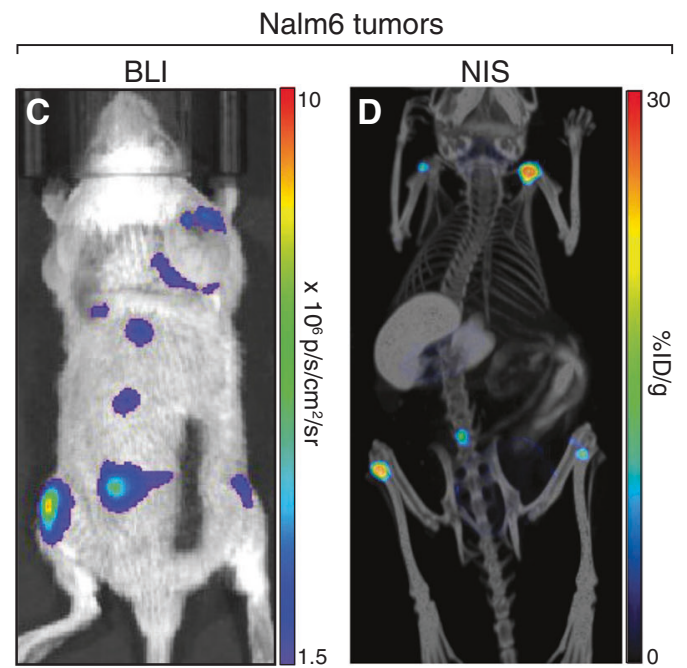

location of bone metastasis) a and 2D z-stack slices of the thoracic region (3-mm interval) b. c, d SCID beige mice were implanted intravenously with Nalm6-Fluc-hNIS cells; a representative mouse is shown. Nalm6-Fluc-hNIS tumors were imaged by BLI on day $26 \mathbf{c}$ and by SPECT/CT using ${ }^{125} \mathrm{I}$ on day $25 \mathrm{~d}$

when the lungs were excised (Fig. 3c). In contrast, in mice implanted with NIS-expressing 4T1-mNIS cells, thoracic, abdominal, bone, and superficial subcutaneous 4T1-mNIS tumors were readily distinguished by PET/CT imaging of NIS (Fig. 3d, e and S2). Thus, compared to BLI, NIS imaging provided additional clarity of tumor burden, tumor location, and individual tumor distribution within the animals.

\section{Noninvasive quantitation of NIS signal correlates strongly to tumor volume}

In pre-clinical imaging studies, reporter signal is frequently used to quantitate tumor burden. However, luciferase and other optical reporters are subject to tissue attenuation, which makes accurate quantitation difficult for non-surface tumors. As radiotracer signal is not attenuated by tissues, we were able to noninvasively quantitate NIS signal in the tumor-bearing mice at all tissue depths. Furthermore, the high-resolution images obtained using NIS imaging facilitated quantification of individual tumor nodules (Fig. 4a). $\left[{ }^{18} \mathrm{~F}\right]$-TFB uptake was similar in all tumor types, though thoracic tumors exhibited slightly higher radiotracer uptake, whereas abdominal tumors exhibited slightly lower radiotracer uptake. Whether these differences have biological significance is unknown.

To determine whether NIS radiotracer uptake accurately predicts tumor volume, we measured NIS reporter signal from subcutaneous tumors. Mice were implanted with 4T1Fluc-Neo/mNIS-Puro cells, which express both luciferase and NIS, in the right hind flank. SPECT/CT imaging was 
A

\section{B Post-mortem}

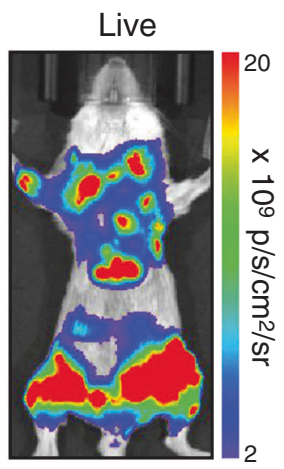

C
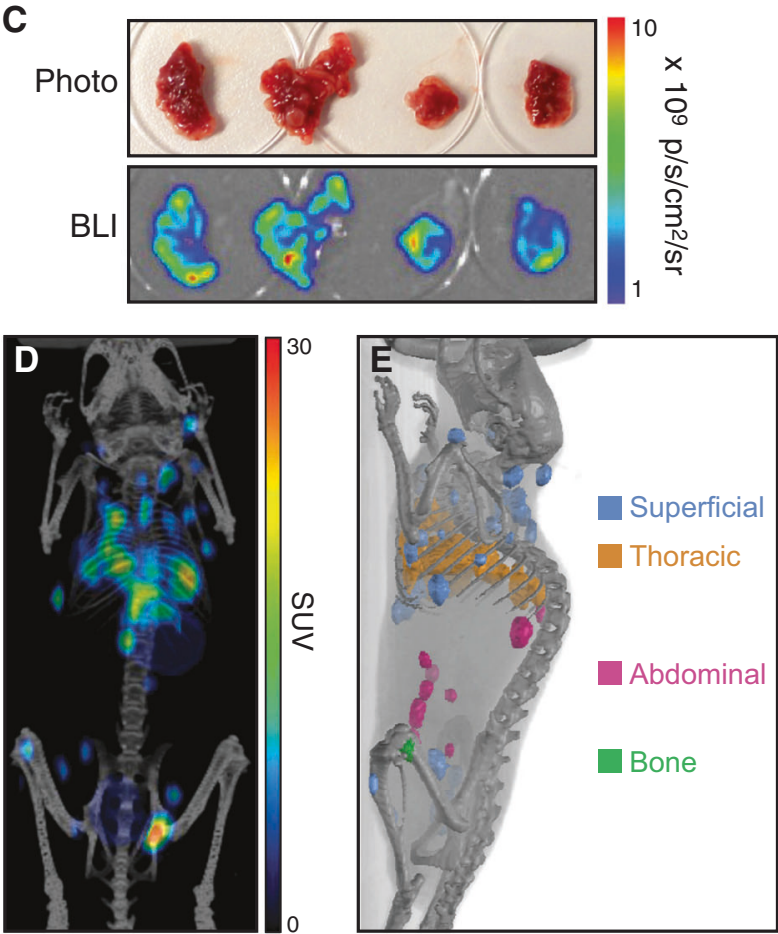

Fig. 3 Discrimination of surface and deep-tissue tumors using NIS. a-c Balb/c mice were implanted intravenously with 4T1-Fluc-Neo cells; a representative mouse is shown. a Tumors were imaged by BLI. b Post-mortem autopsy and BLI was performed upon killing and removal of the skin. c Excised lung lobes were imaged by BLI. d, e Balb/c mice were implanted intravenously with 4T1-mNIS cells and PET/CT imaging was performed after 20 days using $\left[{ }^{18} \mathrm{~F}\right]-\mathrm{TFB}$; a representative mouse is shown. d Global $\left[{ }^{18} \mathrm{~F}\right]$-TFB uptake in mice. e Tomographic representation of tumors from $\mathbf{d}$, colored based on tumor locations

used to measure NIS signal $\left(\left[{ }^{99 \mathrm{~m}} \mathrm{Tc}\right]-\right.$ pertechnetate uptake) from each tumor, whereas calipers and CT were used to calculate tumor volumes. $\left[{ }^{99 \mathrm{~m}} \mathrm{Tc}\right]-$ pertechnetate uptake over the tumor correlated closely with tumor volume (Fig. 4b), demonstrating consistency of NIS-dependent radiotracer uptake over a large range of tumor volumes. We also examined the relationship of luciferase signal to tumor volume using BLI. Fluc activity (total flux) correlated closely with tumor volume (Fig. 4c), consistent with previous
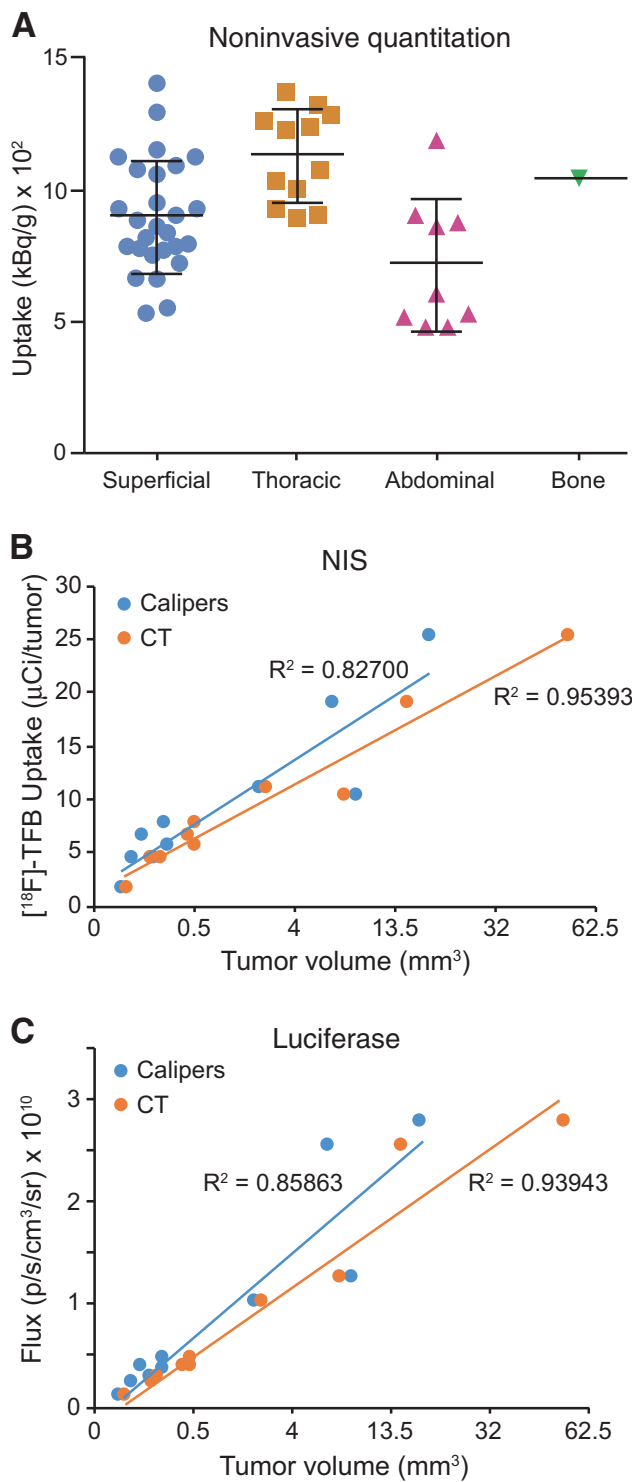

Fig. 4 In vivo correlation of Fluc and NIS signal to tumor volume. a Median uptake $(\mathrm{kBq} / \mathrm{g})$ of $\left[{ }^{18} \mathrm{~F}\right]-\mathrm{TFB}$ from individual $4 \mathrm{~T} 1-\mathrm{mNIS}$ tumors from mouse in Fig. $3 \mathrm{~d}$ and e. b, $\mathbf{c}$ Five SCID beige mice were implanted subcutaneously with 4T1-Fluc-Neo/mNIS-Puro cells. On days 9 and 16 after implantation tumors were measured with calipers and BLI imaging was performed. SPECT/CT imaging using $\left[{ }^{99 \mathrm{~m}} \mathrm{Tc}\right]-$ pertechnetate was performed on days 10 and 17 after implantation. Tumor volumes determined by caliper measurements or CT were compared to [ ${ }^{99 \mathrm{~m}} \mathrm{Tc}$ ]-pertechnetate uptake $\mathbf{b}$ and total tumor flux $\mathbf{c}$

reports that luciferase signal accurately reflects tumor volume for surface tumors $[19,45,46]$.

\section{Combination BLI and NIS imaging offers high sensitivity, resolution, and precise anatomical localization}

Our results demonstrated that NIS imaging of tumors provides several advantages over BLI, including high spatial 


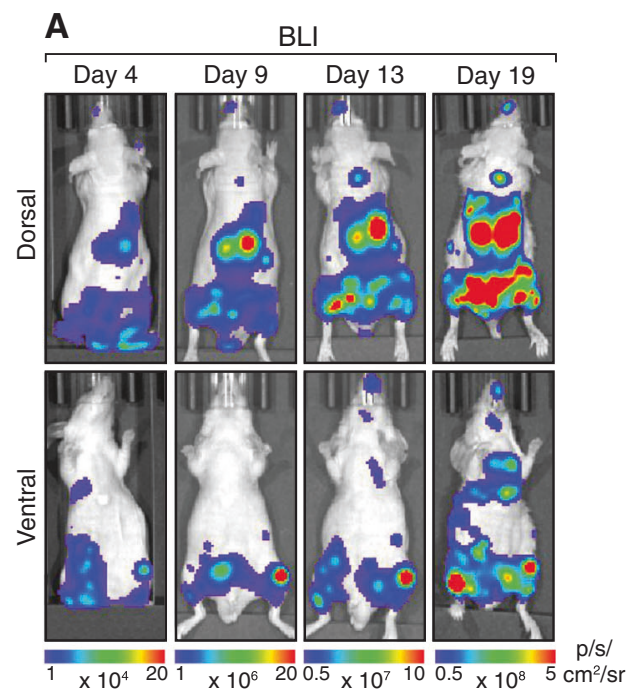

Fig. 5 Multi-modality BLI and NIS imaging of tumors. SCID beige mice were implanted intravenously with 4T1-Fluc-Neo/mNIS-Puro cells; a representative mouse is shown. a Tumors were tracked over

resolution of individual tumor nodules, precise anatomical localization of tumors, and the capacity to more accurately detect and noninvasively quantitate deep-tissue tumors. Nonetheless, BLI offers several benefits over NIS imaging, including its high sensitivity, relative ease-of-use, relative speed, and the lower cost of imaging. Therefore, a multimodality combination of BLI and NIS imaging should represent an enhancement over tumor imaging using either reporter alone. To evaluate combined BLI and NIS imaging, we implanted mice intravenously with 4T1-Fluc-Neo/ mNIS-Puro cells and performed BLI and NIS imaging of the resulting tumors. Using BLI we readily detected tumors early after implantation (Day 4; earliest time examined) (Fig. 5a). Moreover, three mice were imaged (dorsal and ventral) within $10 \mathrm{~min}$, making this a convenient way to quickly asses and monitor tumor burden at earlier time points. When tumor burden became prominent, NIS imaging by PET/CT using $\left[{ }^{18} \mathrm{~F}\right]-\mathrm{TFB}$ afforded high spatial resolution for the discrimination of individual tumor nodules (Fig. 5b and S3), which was not possible by BLI. NIS imaging also facilitated precise anatomical localization and quantification of the tumors. Thus, multi-modality BLI and NIS imaging offers both high sensitivity and resolution, and maintains much of the ease-of-use of BLI while accurately detecting and quantitating deep-tissue tumors.

\section{Discussion}

Noninvasive tracking of tumors through BLI has played an important role in pre-clinical evaluation and development of cancer therapies. Here, we demonstrated that BLI of tumors

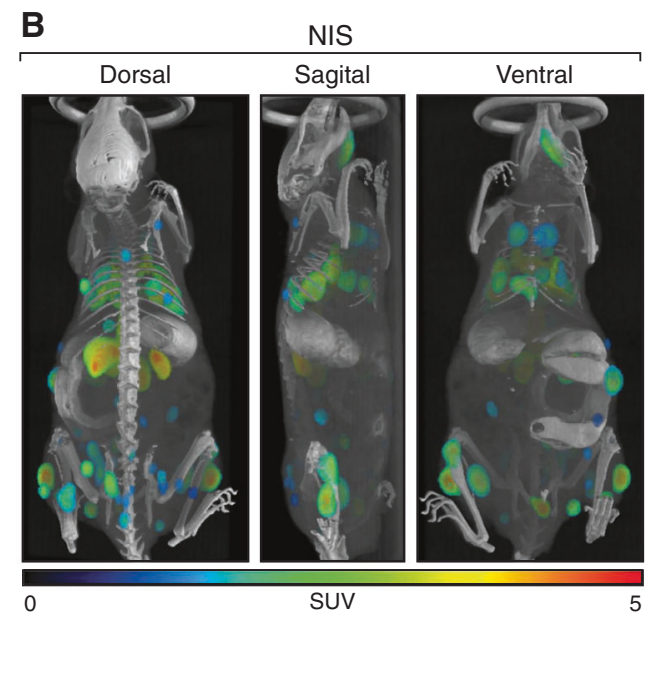

time using BLI. b On day 20 after implantation, PET/CT imaging was performed using $\left[{ }^{18} \mathrm{~F}\right]-\mathrm{TFB}$

can be enhanced by using multi-modality imaging with NIS. As a reporter gene, NIS offers several advantages relative to BLI. NIS imaging by small animal SPECT or PET produces tomographic images with spatial resolution below $1-\mathrm{mm}$ (range $0.9 \mathrm{~mm}$ to $0.16 \mathrm{~mm}$ ), dependent on the scanner and collimator used for image acquisitions [39, 40]. Moreover, when used in combination with $\mathrm{CT}$, precise anatomical localization of signal is possible. NIS-expressing tumors can be readily detected at most anatomical locations, though background NIS signal can limit detection of tumors in the thyroid, salivary glands, mammary glands, and stomach. As radiotracer signal is not subject to tissue attenuation in mice, or can be corrected for attenuation, imaging and noninvasive quantitation of deep-tissue tumors is possible. Compared with other nuclear reporters, NIS also offers more versatility as a reporter gene due to the ready availability of a number of radiotracers for NIS imaging. Thus, NIS imaging is well-suited to complement standard BLI. Indeed, several groups have demonstrated the benefits of combining BLI with imaging of nuclear reporter genes (NIS, SSTR2, HSVTK) for tracking immune cells, regenerative cells, or therapeutic cells in pre-clinical studies [16, 19, 22, 47-51].

Many key questions in pre-clinical oncology research center around tumor growth and metastasis, and the effect of potential therapies on these processes. Noninvasive imaging allows researchers to answer these questions more effectively and accurately in living animals. Tumor growth and the formation of metastases can be tracked in the same animal over time. This tracking facilitates more accurate disease modeling, better equipping researchers to determine when, if at all, to begin treatment studies. Moreover, 
Table 1 Comparison of the strengths and weaknesses of BLI and NIS Imaging

\begin{tabular}{|c|c|c|}
\hline Parameter & BLI & NIS Imaging \\
\hline Equipment & Optical Imager & SPECT or PET \\
\hline Spatial Resolution & Several mm & $\begin{array}{l}\text { Less than } 1 \mathrm{~mm} \text { (dependent on } \\
\text { equipment) }\end{array}$ \\
\hline Sensitivity & $\begin{array}{l}\text { Highest } \\
\text { (less than } 10 \text { cells in vitro) } \\
\text { (depth dependent in vivo) }\end{array}$ & $\begin{array}{l}\text { High } \\
\text { (1000-2000 cells in vitro) } \\
\text { (depth independent in vivo) }\end{array}$ \\
\hline Depth of signal & $\begin{array}{l}\sim 1 \mathrm{~cm} \text { (exponential signal attenuation with } \\
\text { depth) }\end{array}$ & $\begin{array}{l}\text { No limit (minimal signal attenuation with } \\
\text { depth) }\end{array}$ \\
\hline Signal quantitation & Surface-weighted & Accurate at all tissue depths \\
\hline Throughput & $\begin{array}{l}\text { High ( 1-2 min per scan up to } 5 \text { mice } \\
\text { simultaneously) }\end{array}$ & $\begin{array}{l}\text { Low ( 15-20 min per scan 1-4 mice } \\
\text { simultaneously) }\end{array}$ \\
\hline
\end{tabular}

therapy responses can be evaluated in real-time, and residual disease can be easily detected and tracked in the animals. Given the widespread application of noninvasive imaging in the field of oncology, the advancement of methods, such as that described here, for more accurately imaging tumors is highly desirable.

Although BLI offers superior sensitivity to NIS for imaging of superficial tissues, use of the recently developed PET tracer $\left[{ }^{18} \mathrm{~F}\right]-\mathrm{TFB}$ offers increased sensitivity and quantitative accuracy for imaging NIS in deep tissues [38, 52, 53]. Using $\left[{ }^{18} \mathrm{~F}\right]-\mathrm{TFB}$ we detected as few as 1000 NISpositive cells (Fig. 1d). Likewise, Fruhwirth and colleagues were able to detect 2000 NIS-positive cells within a volume of $5 \times 10^{5}$ cells [31]. In our studies, NIS imaging also provided superior sensitivity to $\mathrm{CT}$ imaging alone, which was unable to detect most soft tissue tumors in the tumorbearing mice. Recently, NIS was used to track the fate of therapeutic CAR-T cells in mice [21]. Moreover, because NIS is directly clinically translatable and has therapeutic potential itself [54-58], the use of NIS for tracking therapeutic cells both pre-clinically and clinically is likely to rise in the future. For this application, advances in the sensitivity of NIS imaging will provide a clear advantage. Although advances may occur in the form of further radiotracer development or more sensitive equipment, modulation of NIS itself may also contribute to increased sensitivity. Because sensitivity is directly related to NIS or luciferase expression and activity within the cells, sensitivity can be improved by increasing protein expression, or increasing enzyme or transporter activity.

Just as advances have been made in NIS imaging, new technologies have improved signal quantitation and localization by BLI. Newer optical imagers (e.g., the Perkin Elmer IVIS ${ }^{\oplus}$ SpectrumCT, and Bruker InVivo X-treme II), combine BLI with CT or X-ray imaging to improve anatomical localization of signal. The development of more advanced computer algorithms has also increased the accuracy of BLI signal quantification by accounting more for signal depths. Despite these advances, however, signal attenuation still prevents precise anatomical localization and accurate quantitation of deep-tissue tumors by BLI alone.

Combining BLI with NIS imaging exploits the strengths of both imaging technologies (Table 1). BLI can be used for sensitive and relatively easy monitoring of tumor formation and growth. NIS imaging at key intervals provides tomographic, high-resolution images that are fully quantitative, and anatomically localized. Moreover, by combining modalities in a single animal, this superior clarity can be achieved using fewer animals, saving time and money while accelerating therapy evaluation.

Acknowledgements We thank the Imanis team and Teresa Decklever (Small Animal Radiology Imaging Core Facility, Mayo Clinic) for technical assistance in performing BLI and PET/SPECT imaging.

Funding These studies were supported from the Research and Development budget of Imanis Life Sciences. SK was supported by a TRF and Mahidol University (Thai Royal Golden Jubilee) PhD Scholarship.

\section{Compliance with ethical standards}

Conflict of interest RV, LS, KWP are employees of Imanis. KW, SJR, Imanis Life Sciences, and Mayo Clinic have a financial interest in the NIS technology used in this paper. The authors declare that they have no conflict of interest.

Publisher's note: Springer Nature remains neutral with regard to jurisdictional claims in published maps and institutional affiliations.

Open Access This article is licensed under a Creative Commons Attribution 4.0 International License, which permits use, sharing, adaptation, distribution and reproduction in any medium or format, as long as you give appropriate credit to the original author(s) and the source, provide a link to the Creative Commons license, and indicate if changes were made. The images or other third party material in this article are included in the article's Creative Commons license, unless indicated otherwise in a credit line to the material. If material is not included in the article's Creative Commons license and your intended use is not permitted by statutory regulation or exceeds the permitted use, you will need to obtain permission directly from the copyright holder. To view a copy of this license, visit http://creativecommons. org/licenses/by/4.0/. 


\section{References}

1. Wang Y, Tseng JC, Sun Y, Beck AH, Kung AL. Noninvasive imaging of tumor burden and molecular pathways in mouse models of cancer. Cold Spring Harb Protoc. 2015;2015:135-44.

2. James ML, Gambhir SS. A molecular imaging primer: modalities, imaging agents, and applications. Physiol Rev. 2012;92:897-965.

3. O'Neill K, Lyons SK, Gallagher WM, Curran KM, Byrne AT. Bioluminescent imaging: a critical tool in pre-clinical oncology research. J Pathol. 2010;220:317-27.

4. Baker M. Whole-animal imaging: the whole picture. Nature. 2010;463:977-80.

5. Kim JE, Kalimuthu S, Ahn BC. In vivo cell tracking with bioluminescence imaging. Nucl Med Mol Imaging. 2015;49:3-10.

6. Spitzweg C, Joba W, Eisenmenger W, Heufelder AE. Analysis of human sodium iodide symporter gene expression in extrathyroidal tissues and cloning of its complementary deoxyribonucleic acids from salivary gland, mammary gland, and gastric mucosa. J Clin Endocrinol Metab. 1998;83:1746-51.

7. Smanik PA, Liu Q, Furminger TL, Ryu K, Xing S, Mazzaferri EL, et al. Cloning of the human sodium lodide symporter. Biochem Biophys Res Commun. 1996;226:339-45.

8. Dai G, Levy O, Carrasco N. Cloning and characterization of the thyroid iodide transporter. Nature. 1996;379:458-60.

9. Dadachova E, Carrasco N. The $\mathrm{Na} / \mathrm{I}$ symporter (NIS): imaging and therapeutic applications. Semin Nucl Med. 2004;34:23-31.

10. Portulano C, Paroder-Belenitsky M, Carrasco N. The Na+/Isymporter (NIS): mechanism and medical impact. Endocr Rev. 2014;35:106-49.

11. Penheiter AR, Russell SJ, Carlson SK. The sodium iodide symporter (NIS) as an imaging reporter for gene, viral, and cell-based therapies. Curr Gene Ther. 2012;12:33-47.

12. Shimura H, Haraguchi K, Miyazaki A, Endo T, Onaya T. Iodide uptake and experimental 131I therapy in transplanted undifferentiated thyroid cancer cells expressing the $\mathrm{Na}+/ \mathrm{I}-$ symporter gene. Endocrinology. 1997;138:4493-6.

13. Miller A, Russell SJ. The use of the NIS reporter gene for optimizing oncolytic virotherapy. Expert Opin Biol Ther. 2016;16:15-32.

14. Hickey RD, Mao SA, Amiot B, Suksanpaisan L, Miller A, Nace $\mathrm{R}$, et al. Noninvasive 3-dimensional imaging of liver regeneration in a mouse model of hereditary tyrosinemia type 1 using the sodium iodide symporter gene. Liver Transpl. 2015;21:442-53.

15. Hickey RD, Mao SA, Glorioso J, Elgilani F, Amiot B, Chen H, et al. Curative ex vivo liver-directed gene therapy in a pig model of hereditary tyrosinemia type 1. Sci Transl Med. 2016;8:349ra99.

16. Holvoet B, Quattrocelli M, Belderbos S, Pollaris L, Wolfs E, Gheysens O, et al. Sodium iodide symporter PET and BLI noninvasively reveal mesoangioblast survival in dystrophic mice. Stem Cell Rep. 2015;5:1183-95.

17. Lee AR, Woo SK, Kang SK, Lee SY, Lee MY, Park NW, et al. Adenovirus-mediated expression of human sodium-iodide symporter gene permits in vivo tracking of adipose tissue-derived stem cells in a canine myocardial infarction model. Nucl Med Biol. 2015;42:621-9.

18. Terrovitis J, Kwok KF, Lautamaki R, Engles JM, Barth AS, Kizana E, et al. Ectopic expression of the sodium-iodide symporter enables imaging of transplanted cardiac stem cells in vivo by single-photon emission computed tomography or positron emission tomography. J Am Coll Cardiol. 2008;52:1652-60.

19. Wolfs E, Holvoet B, Gijsbers R, Casteels C, Roberts SJ, Struys T, et al. Optimization of multimodal imaging of mesenchymal stem cells using the human sodium iodide symporter for PET and Cerenkov luminescence imaging. PLoS ONEe. 2014;9:e94833.
20. Dwyer RM, Ryan J, Havelin RJ, Morris JC, Miller BW, Liu Z, et al. Mesenchymal Stem Cell-mediated delivery of the sodium iodide symporter supports radionuclide imaging and treatment of breast cancer. Stem Cells. 2011;29:1149-57.

21. Emami-Shahri N, Foster J, Kashani R, Gazinska P, Cook C, Sosabowski J, et al. Clinically compliant spatial and temporal imaging of chimeric antigen receptor T-cells. Nat Commun. 2018;9:1081.

22. Lee HW, Jeon YH, Hwang MH, Kim JE, Park TI, Ha JH, et al. Dual reporter gene imaging for tracking macrophage migration using the human sodium iodide symporter and an enhanced firefly luciferase in a murine inflammation model. Mol Imaging Biol. 2013;15:703-12.

23. Lee HW, Yoon SY, Singh TD, Choi YJ, Lee HJ, Park JY, et al. Tracking of dendritic cell migration into lymph nodes using molecular imaging with sodium iodide symporter and enhanced firefly luciferase genes. Sci Rep. 2015;5:9865.

24. Seo JH, Jeon YH, Lee YJ, Yoon GS, Won DI, Ha JH, et al. Trafficking macrophage migration using reporter gene imaging with human sodium iodide symporter in animal models of inflammation. J Nucl Med. 2010;51:1637-43.

25. Moulay G, Ohtani T, Ogut O, Guenzel A, Behfar A, Zakeri R, et al. Cardiac AAV9 gene delivery strategies in adult canines: assessment by long-term serial SPECT imaging of sodium iodide symporter expression. Mol Ther. 2015;23:1211-21.

26. Wang J, Arulanandam R, Wassenaar R, Falls T, Petryk J, Paget J, et al. Enhancing expression of functional human sodium iodide symporter and somatostatin receptor in recombinant oncolytic vaccinia virus for in vivo imaging of tumors. J Nucl Med. 2017;58:221-7.

27. Carlson SK, Classic KL, Hadac EM, Dingli D, Bender CE, Kemp $\mathrm{BJ}$, et al. Quantitative molecular imaging of viral therapy for pancreatic cancer using an engineered measles virus expressing the sodium-iodide symporter reporter gene. AJR Am J Roentgenol. 2009;192:279-87.

28. Goel A, Carlson SK, Classic KL, Greiner S, Naik S, Power AT, et al. Radioiodide imaging and radiovirotherapy of multiple myeloma using VSV(Delta51)-NIS, an attenuated vesicular stomatitis virus encoding the sodium iodide symporter gene. Blood. 2007; 110:2342-50.

29. Mansfield DC, Kyula JN, Rosenfelder N, Chao-Chu J, KramerMarek G, Khan AA, et al. Oncolytic vaccinia virus as a vector for therapeutic sodium iodide symporter gene therapy in prostate cancer. Gene Ther. 2016;23:357-68.

30. Msaouel P, Iankov ID, Allen C, Aderca I, Federspiel MJ, Tindall DJ, et al. Noninvasive imaging and radiovirotherapy of prostate cancer using an oncolytic measles virus expressing the sodium iodide symporter. Mol Ther. 2009;17:2041-8.

31. Diocou S, Volpe A, Jauregui-Osoro M, Boudjemeline $M$, Chuamsaamarkkee K, Man F, et al. [(18)F]tetrafluoroborate-PET/ $\mathrm{CT}$ enables sensitive tumor and metastasis in vivo imaging in a sodium iodide symporter-expressing tumor model. Sci Rep. 2017;7:946.

32. Fruhwirth GO, Diocou S, Blower PJ, Ng T, Mullen GE. A wholebody dual-modality radionuclide optical strategy for preclinical imaging of metastasis and heterogeneous treatment response in different microenvironments. J Nucl Med. 2014;55:686-94.

33. Volpe A, Man F, Lim L, Khoshnevisan A, Blower J, Blower PJ, et alls. J Vis Exp. 2018:e57088.

34. Michigami T, Hiraga T, Williams PJ, Niewolna M, Nishimura R, Mundy GR, et al. The effect of the bisphosphonate ibandronate on breast cancer metastasis to visceral organs. Breast Cancer Res Treat. 2002;75:249-58.

35. Pulaski BA, Ostrand-Rosenberg S. Reduction of established spontaneous mammary carcinoma metastases following immunotherapy with major histocompatibility complex class II 
and B7.1 cell-based tumor vaccines. Cancer Res. 1998;58: 1486-93.

36. Vastenhouw B, Beekman F. Submillimeter total-body murine imaging with U-SPECT-I. J Nucl Med. 2007;48:487-93.

37. Branderhorst W, Vastenhouw B, Beekman FJ. Pixel-based subsets for rapid multi-pinhole SPECT reconstruction. Phys Med Biol. 2010;55:2023-34.

38. Jiang H, Bansal A, Pandey MK, Peng KW, Suksanpaisan L, Russell SJ, et al. Synthesis of 18 F-tetrafluoroborate via radiofluorination of boron trifluoride and evaluation in a murine C6glioma tumor model. J Nucl Med. 2016;57:1454-9.

39. Deleye S, Van Holen R, Verhaeghe J, Vandenberghe S, Stroobants S, Staelens S. Performance evaluation of small-animal multipinhole muSPECT scanners for mouse imaging. Eur J Nucl Med Mol Imaging. 2013;40:744-58.

40. Nagy K, Toth M, Major P, Patay G, Egri G, Haggkvist J, et al. Performance evaluation of the small-animal nanoScan PET/MRI system. J Nucl Med. 2013;54:1825-32.

41. Barrett DM, Seif AE, Carpenito C, Teachey DT, Fish JD, June $\mathrm{CH}$, et al. Noninvasive bioluminescent imaging of primary patient acute lymphoblastic leukemia: a strategy for preclinical modeling. Blood. 2011;118:e112-7.

42. Benito J, Shi Y, Szymanska B, Carol H, Boehm I, Lu H, et al. Pronounced hypoxia in models of murine and human leukemia: high efficacy of hypoxia-activated prodrug PR-104. PLoS ONE. 2011;6:e23108.

43. Brentjens RJ, Santos E, Nikhamin Y, Yeh R, Matsushita M, La Perle K, et al. Genetically targeted T cells eradicate systemic acute lymphoblastic leukemia xenografts. Clin Cancer Res. 2007;13(18 Pt 1):5426-35.

44. Santos EB, Yeh R, Lee J, Nikhamin Y, Punzalan B, Punzalan B, et al. Sensitive in vivo imaging of $T$ cells using a membranebound Gaussia princeps luciferase. Nat Med. 2009;15:338-44.

45. Chien YC, Chen JC, Lin WC, Ding HJ, Wang HE, Kao $\mathrm{CH}$, et al. Using [18F]FBAU for imaging brain tumor progression in an F98/tk-luc glioma-bearing rat model. Oncol Rep. 2014;32:691-9.

46. Ma X, Liu Z, Yang X, Gao Q, Zhu S, Qin C, et al. Dual-modality monitoring of tumor response to cyclophosphamide therapy in mice with bioluminescence imaging and small-animal positron emission tomography. Mol Imaging. 2011;10:278-83.

47. Love Z, Wang F, Dennis J, Awadallah A, Salem N, Lin Y, et al. Imaging of mesenchymal stem cell transplant by bioluminescence and PET. J Nucl Med. 2007;48:2011-20.
48. Neyrinck K, Breuls N, Holvoet B, Oosterlinck W, Wolfs E, Vanbilloen $\mathrm{H}$, et al. The human somatostatin receptor type 2 as an imaging and suicide reporter gene for pluripotent stem cellderived therapy of myocardial infarction. Theranostics. 2018;8:2799-813.

49. Pei Z, Lan X, Cheng Z, Qin C, Wang P, He Y, et al. A multimodality reporter gene for monitoring transplanted stem cells. Nucl Med Biol. 2012;39:813-20.

50. Pei Z, Lan X, Cheng Z, Qin C, Xia X, Yuan H, et al. Multimodality molecular imaging to monitor transplanted stem cells for the treatment of ischemic heart disease. PLoS ONEe. 2014;9:e90543.

51. Sun $\mathrm{N}$, Lee A, Wu JC. Long term non-invasive imaging of embryonic stem cells using reporter genes. Nat Protoc. 2009;4:1192-201.

52. Jauregui-Osoro M, Sunassee K, Weeks AJ, Berry DJ, Paul RL, Cleij M, et al. Synthesis and biological evaluation of [(18)F]tetrafluoroborate: a PET imaging agent for thyroid disease and reporter gene imaging of the sodium/iodide symporter. Eur J Nucl Med Mol Imaging. 2010;37:2108-16.

53. Weeks AJ, Jauregui-Osoro M, Cleij M, Blower JE, Ballinger JR, Blower PJ. Evaluation of $[18 \mathrm{~F}]$-tetrafluoroborate as a potential PET imaging agent for the human sodium/iodide symporter in a new colon carcinoma cell line, HCT116, expressing hNIS. Nucl Med Commun. 2011;32:98-105.

54. Dwyer RM, Bergert ER, O'Connor MK, Gendler SJ, Morris JC. In vivo radioiodide imaging and treatment of breast cancer xenografts after MUC1-driven expression of the sodium iodide symporter. Clin Cancer Res. 2005;11:1483-9.

55. Hutzen B, Pierson CR, Russell SJ, Galanis E, Raffel C, Studebaker $\mathrm{AW}$. Treatment of medulloblastoma using an oncolytic measles virus encoding the thyroidal sodium iodide symporter shows enhanced efficacy with radioiodine. BMC Cancer. 2012;12:508.

56. Knoop K, Schwenk N, Schmohl K, Muller A, Zach C, Cyran C, et al. Mesenchymal stem cell-mediated, tumor stroma-targeted radioiodine therapy of metastatic colon cancer using the sodium iodide symporter as theranostic gene. J Nucl Med. 2015;56:600-6.

57. Renier C, Do J, Reyna-Neyra A, Foster D, De A, Vogel H, et al. Regression of experimental NIS-expressing breast cancer brain metastases in response to radioiodide/gemcitabine dual therapy. Oncotarget. 2016;7:54811-24.

58. Schmohl KA, Gupta A, Grunwald GK, Trajkovic-Arsic M, Klutz $\mathrm{K}$, Braren $\mathrm{R}$, et al. Imaging and targeted therapy of pancreatic ductal adenocarcinoma using the theranostic sodium iodide symporter (NIS) gene. Oncotarget. 2017;8:33393-404. 